\title{
Il sistema della memoria ne La Luna e i falò
}

\author{
Giovanni Albertocchi \\ Universitat de Girona \\ giovanni.albertocchi@udg.edu
}

\begin{abstract}
Riassunto
Il saggio studia il ruolo determinante che il viaggio, di partenza e di ritorno, svolge all'interno de La luna e i falò, l'ultimo romanzo di Cesare Pavese. Grazie a questo doppio movimento di andata e ritorno, presente in gran parte dell'opera pavesiana, questo lavoro mette in rilievo come il protagonista del romanzo non sarà mai in grado di ritornare nello spazio delle origini. Sarà solo capace di attivare tutta la forza evocativa della memoria, fino a raggiungere la poesia, lirica, tragica ed elegiaca, inerente all'impossibile e malinconica incapacità di ritornare nel proprio mondo passato.
\end{abstract}

Palabras clave: Ritorno, origini, infanzia, memoria, passato, Pavese, La Luna e i falò.

The memory system in La luna e i falo.

\begin{abstract}
The essay studies the dominant role of the return jorney in La luna e $i$ falo, the last novel of Cesare Pavese. Thanks to this double mouvement (going and returning), present in the Pavesian work, this article demostrates that the principal characters of Pavese cannot return to their original space. They can only look at the childhood scenaries through the evocative memory. They reach in this way the lyric, tragic and elegiac poetry. It consents them to look at the melancholic world of their own past time.
\end{abstract}

Key words: Return, origins, childhood, memory, past, Pavese, La Luna e i falò.

Albertocchi, Giovanni. 2011. Il sistema della memoria nella Luna e i falò. Cuadernos de Filología Italiana, $\mathrm{n}^{\circ}$ extraordinario: págs. 21-32.

L'8 luglio 1950, Cesare Pavese tornava per l'ultima volta a Santo Stefano Belbo, probabilmente per dare l'addio all'amico del cuore, Pinolo Scaglione, ed ai luoghi dell'infanzia. A Pinolo ha portato una copia del romanzo appena uscito, La Luna e $i$ 
falo, con una dedica che, come ricorda Franco Vaccaneo (Vaccaneo 1999: 93), lo lasciò piuttosto interdetto: «A Pinolo questo libro - l'ultimo che avrò mai scritto». Vedendolo preoccupato, Cesare prende la penna in mano e aggiunge un «forse» («forse l'ultimo») per attenuare quella formula che non faceva presagire nulla di buono.

La luna e i falò, così come aveva preannunciato l'autore sarà effettivamente il suo ultimo romanzo. L'idea di scriverlo gli era venuta nell'estate del 1949. In una lettera ai coniugi Varigotti, del 17 luglio, confessava con entusiamo: «Io sono come pazzo perché ho avuto una grande intuizione - quasi una mirabile visione (naturalmente di stalle, sudore, contadinotti, verderame e letame ecc.) su cui dovrei costruire una modesta Divina commedia ${ }^{1}$ (Pavese 1968: II, 659). Il 18 settembre inizia la stesura del romanzo, che scrive di getto, in meno di due mesi, con una rapidità insolita anche per uno scrittore come lui abituato a ritmi sostenuti. «9 novembre. -leggiamo in una nota del Mestiere di vivere - Finito La luna e i falò. Dal 18 sett. sono meno di due mesi. Quasi sempre un capitolo al giorno. È certo l'exploit piú forte sinora» (Pavese 2000: 375).

Il manoscritto del romanzo, come riferisce Claudio Sensi, rivela in effetti una stesura ben articolata e già definitiva, soprattutto nelle parti dedicate alla memoria, senza modificazioni o incertezze, come se Pavese avesse trasferito sulla pagina una storia già immaginata e pronta nella sua fantasia ${ }^{2}$.

La critica è concorde nel considerare il romanzo il punto di arrivo di diversi temi e motivi che attraversano l'intera produzione dello scrittore. Il più importante è quello del «ritorno» o del «reduce» che era apparso per la prima volta in una poesia famosissima, I mari del Sud, con cui si apre la raccolta Lavorare stanca. La voce poetante è affidata ad un adolescente che si inerpica su una collina delle Langhe con il cugino appena rientrato in paese dopo essere stato per vent'anni «in giro per il mondo» ed aver fatto mille mestieri.

Il tema del ritorno lo troviamo anche ne La Langa, uno dei racconti di Feria d'agosto. Il protagonista torna al paese delle Langhe dopo aver fatto fortuna all'estero. Vorrebbe sposare una ragazza del posto, ma poi ci rinuncia perché -confessa«se mi fossi portata dietro in città una di quelle ragazze [...] avrei avuto il mio paese in casa e non avrei mai più potuto ricordarmelo come adesso me n'era tornato il gusto» (Pavese 1968: 18). Alla fine decide di andarsene un'altra volta, per sempre.

C'è già in nuce il senso del fallimento che proverà anche Anguilla, ne La luna e i falò: il paese è meglio ricordarlo che rivederlo. Il ritorno implica lo smacco esistenziale per non ritrovare più il passato, perché lo si è perduto per sempre. Mentre

1 Il riferimento alla Divina Commedia è assai pertinente. Secondo Stefano Gioanardi, il modello dantesco appare non solo nel rapporto fra Nuto e Anguilla che richiama quello di Virgilio e Dante, ma anche «nello schema degli «incontri» compiuti da Anguilla, con o senza il suo compagno, con personaggi che appaiono, rendono edotto il lettore sulla loro condizione e poi scompaiono [...]» (Gioanardi 1996: 641).

2 Claudio Sensi, nelle «Notizie sul testo» ci dice: «la stesura manoscritta rivela una sicurezza mirabile nell'affidare alla scrittura un organismo narrativo perfettamente articolato nei suoi snodi, calibrato, bilanciato, disteso nella quiete disinvolta di ciò che nasce come praticamente definitivo» (Pavese 2000: 1085). 
uno sta lontano, i luoghi e le persone cambiano, invecchiano, scompaiono. Solo la memoria li mantiene in vita. Ma perché l'incantesimo funzioni occorre sacrificare tutto, andarsene dai luoghi in cui si è vissuto, che così rimarranno per sempre intatti nel ricordo.

Pure fallimentare è il ritorno a Torino di Clelia, la protagonista di Tra donne sole, che Italo Calvino, all'epoca in cui lavoravano entrambi all'Einaudi, aveva definito ironicamente come la controfigura di Pavese «con la parrucca e i seni finti». Clelia ha fatto fortuna a Roma, nell'ambiente dell'alta moda: crede che tornando a Torino la memoria la risarcirà di una vita di sacrifici, ma tutto è cambiato e lei si ritrova a chiedersi amaramente «se val la pena darsi da fare per arrivare dov'ero arrivata, e non essere più niente» (Pavese 2000: 738).

Chi ritorna lo fa perché oppresso dalla nostalgia e desidera rivedere le persone ed i luoghi da cui si è allontanato. In realtà ha bisogno di tornare per verificare in loco l'attendibilità della memoria che ha congelato per tanti anni quelle antiche immagini.

Cesare Pavese ha già elaborato, nel momento in cui si accinge alla stesura del romanzo, una sua personale teoria della rimembranza che deve molto a Giacomo Leopardi, ma anche a Carl Gustav Jung ed a Sigmund Freud. L'area privilegiata della memoria è naturalmente l'infanzia: è questa l'epoca in cui la natura imprime per la prima volta nell'animo del bambino gli «stampi», gli archetipi direbbe Jung, ossia «le immagini primordiali» delle cose che vede, «come a dire - aveva dichiarato lo scrittore in una lettera del 27 giugno 1942 a Fernanda Pivano - l'albero, la casa, la vite, il sentiero, la sera, il pane, la frutta, ecc.» (Pavese 1968: II, 425). Ma alla visione delle cose non corrisponde una forma di conoscenza delle stesse: vedere non è infatti capire, perché la ricezione nel bambino è alterata o manomessa dall'emozione che archivia i dati secondo codici irrazionali. Bisogna quindi tornare a rivedere le stesse cose da adulto, per recuperarne il senso che allora ci era sfuggito. «Le cose -leggiamo nel Mestiere di vivere-si scoprono attraverso i ricordi che se ne hanno. Ricordare una cosa significa vederla -ora soltanto-per la prima volta» (Pavese 2000: 232). «Bisogna sapere - precisa ancora in Stato di grazia, appartenente a Feria d'agosto - che noi non vediamo mai le cose una prima volta, ma sempre la seconda. Allora le scopriamo e insieme le ricordiamo» (Pavese 1968: 145). Il ricordo e la memoria sono quindi una forma di conoscenza: si recupera il passato avvolto nelle nebbie del tempo, lo si capisce e nello stesso tempo si gode di quella singolare vibrazione che prende chiunque si addentri nei meandri del tempo. La memoria dispone così di un doppio registro, quello poetico che ha a che vedere con le emozioni e quello razionale-conoscitivo che se ne appropria, trasformandole in patrimonio esistenziale. Pavese arriva addirittura alla «bizzarria» - come afferma in un altro appunto del Mestiere di vivere - di attribuire alla memoria la capacità di «modificare il proprio passato», «modificandone - cioè scoprendo il significato» (Pavese 2000: 281). Sono riflessioni a cui Sigmund Freud darebbe senza dubbio il nulla osta, enfatizzando semmai l'aspetto terapeutico dell'operazione. Prima di Freud e di Pavese, anche Giacomo Leopardi aveva intuito quella singolare peculiarità della memoria che modifica il passato, nel suo caso specifico gli episodi più tristi, rendendo, come dice nello Zibaldone, «piacevoli [...] anche le ricordanze d'immagini e 
di cose che nella fanciullezza ci erano dolorose, o spaventose» (Leopardi 1991: I, 1122).

Il passato sarebbe infatti uno sterile deposito di avvenimenti che soltanto la memoria può riscattare dalla loro inerzia capendoli e modificandoli all'infinito: «[...] chi sa in quanti modi diversi vedrò ancora il mio passato -conclude Pavese-, e cioè vi scoprirò avvenimenti insospettati» (Pavese 2000: 281).

Lo «stato di grazia» che vive l'adulto nel riconoscere le cose dell'infanzia è probabilmente il punto in cui Pavese si avvicina maggiormente a Leopardi, come dimostra questa riflessione del poeta di Recanati nei riguardi del ricordo: «La sensazione presente non deriva immediatamente dalle cose, non è un'immagine degli oggetti, ma della immagine fanciullesca; una ricordanza, una ripetizione, una ripercussione o riflesso della immagine antica» (Leopardi 1991: I, 366).

In un bellissimo racconto di Feria d'agosto, La vigna, Pavese descrive un adulto di fronte alla vigna che veniva ad osservare da ragazzo. Per un attimo essa diventa una «porta magica» che si apre sul tempo: l'uomo ed il ragazzo si incontrano, come in una specie di Aleph borgesiano. «L'uomo e il ragazzo s'incontrano e sanno e si dicono che il tempo è sfumato». Il rivedere la vigna apporta conoscenza ed emozione, ma l'incantesimo non è ancora finito: «Davanti al sentiero che sale all'orizzonte, l'uomo non ritorna ragazzo: è ragazzo. Per un attimo, in cui giunge a far tacere ogni ricordo, si trova entro gli occhi la vigna immobile, istintiva, immutabile, quale ha sempre saputo di avere nel cuore» (Pavese 1968: 155).

Ma per ricordare e quindi capire, bisogna staccarsi dai luoghi familiari: non basta crescere -a questo ci pensa la vita-, bisogna soprattutto andarsene lontano: «La vita va vissuta lontano dal paese» aveva avvertito il protagonista de I mari del Sud (Pavese 1968: 9). Così si possono capire le ragioni per cui Anguilla ritorna a Santo Stefano Belbo, dopo vent'anni trascorsi a girovagare per gli Stati Uniti: «Potevo spiegare a qualcuno che quel che cercavo era soltanto di vedere qualcosa che avevo già visto?» (Pavese 2000: 814). Anguilla, però, scoprirà anche l'altra faccia della medaglia. Ricordare non è infatti un'operazione inoffensiva: rivedere le cose non vuol dire soltanto capirle, ma anche perderle per sempre. Anguilla è una specie di Orfeo piemontese che si gira indietro per vedere il passato, suscitando così l'ira degli dei che aprono alle sue spalle uno scenario infestato da cadaveri e da assenze. L'Orfeo pavesiano, è bene precisare, è più interessato al proprio passato che al riscatto della moglie Euridice: nei Dialoghi con Leucò aveva detto esplicitamente di essere sceso all'Inferno per cercare «un passato che Euridice non sa»: «ho cercato me stesso - aggiunge -. Non si cerca che questo» (Pavese 1999: 77-78).

Del protagonista del romanzo sappiamo soltanto il soprannome, "Anguilla", che gli è stato affibbiato a causa della sua magrezza. Da bambino è stato "adottato" come «servitore di campagna»: in quell'epoca infatti lo stato passava un piccolo contributo a chi si facesse carico di un trovatello. Il fatto che Pavese scegliesse un «bastardo» come alter ego la dice lunga sul senso di sradicamento e di solitudine da cui si sentiva afflitto.

Dopo vent'anni trascorsi negli Stati Uniti girovagando qua e là, un po' come i personaggi on the road dei romanzi americani che Pavese andava traducendo in italiano, e svolgendo i mestieri più disparati, manovale, barista, trafficante di liquori 
all'epoca del proibizionismo, ecc., Anguilla decide di tornare al paese. Ha anche messo da parte un discreto gruzzolo che gli consente di vivere agiatamente: insomma, lui che se ne era andato da bastardo, può tornare a testa alta e fare sfoggio del benessere acquisito.

Nell'incipit magistrale del romanzo che, va detto subito, si può assaporare come un lungo poema o meglio come un canzoniere in prosa (Giovanardi 1996: 635), il protagonista mette subito in chiaro che non ritorna per ritrovare le propie radici: «C'è una ragione perché sono tornato in questo paese, qui e non invece a Canelli, a Barbaresco o in Alba. Qui non ci sono nato, è quasi certo; dove son nato non lo so; non c'è da queste parti una casa né un pezzo di terra né delle ossa ch'io possa dire "Ecco cos'ero prima di nascere"» (Pavese 2000: 781).

Il fatto di non esservi nato a Santo Stefano Belbo è insomma il requisito che sembra garantire un viaggio più proficuo nel tempo: Anguilla non torna per cercare $\mathrm{i}$ genitori, ma per rivedere e conoscere il proprio passato. Le sue radici non sono quelle biologiche, inesistenti come ha appena dichiarato, ma quelle che la memoria costruirà sulla consapevolezza del proprio passato e del destino che già allora, a sua insaputa, lo spingeva lontano dal paese. Sulla propria genealogia, Anguilla ne sentirà delle belle: secondo Teresa, la ragazza che frequenterà a Genova durante il servizio militare, i suoi «peli ricci» proverebbero che è figlio di una zingara; più pittoresca l'ipotesi di Emilia, una delle inservienti della Mora, che lo fa addirittura «figlio di un saltimbanco e di una capra dell'alta Langa», al che Anguilla ribatte divertito che lui invece si sentiva figlio di un prete. Nuto, una volta che l'amico scherzava sul fatto che ora con i quattrini di mezzo magari «salterà fuori anche mio padre», gli dice di non preoccuparsi perché in fondo «Tuo padre, [...] sei tu».

Un breve inciso sulle ripercussioni e le inevitabili curiosità che provocano in Anguilla le incerte origini familiari, lo troviamo in uno dei capitoli americani del romanzo. Anguilla è a Fresno, in California: ha una ragazza, Rosanne, che è una bastarda come lui, perché in America, precisa, lo sono quasi tutti, «mai che capissi dove avessero padre, madre e la loro terra». A volte pensa che fare un figlio con Rosanne sarebbe un modo per avere un'idea di chi fosse suo padre: «Sarebbe bella, pensavo, se mio figlio somigliasse a mio padre, a mio nonno, e così mi vedessi davanti finalmente chi sono» (Pavese 2000: 855). È in fondo la stessa ragione per cui Anguilla è tornato a Santo Stefano Belbo: per scrutare il paese come se fosse uno specchio in cui veder riflessa la sua immagine e capire finalmente chi fosse.

Anguilla è una specie di Dante che viaggia, come disse Italo Calvino (Calvino 2007: I, 1232), in compagnia di un «rustico» Virgilio, l'amico Nuto, nel proprio passato. Nuto, oltre a rievocare con lui i vecchi tempi, lo aggiorna su quanto è successo in paese durante la sua assenza.

Il presente da cui muove la narrazione è un'area temporale promiscua, nel senso che in essa si confondono i due momenti del ritorno: il ritorno dall'America, avvenuto circa un anno prima, durante il quale il protagonista da Genova, dove si è sistemato, fa diverse puntate a Santo Stefano Belbo, ed il soggiorno ininterrotto di una quindicina di giorni, in agosto, che è lo spazio diegetico e mnemonico più operativo.

Come ha giustamente rilevato Bart Van Den Bossche, ai due spazi cronologici corrispondono due voci narrative, corrispondenti all'Anguilla tornato un anno prima 
dall'America ed all'Anguilla che parla e ricorda durante il soggiorno estivo (Van Den Bossche, 1989: 723). Le due voci si integrano e si confondono in un'attività memorativa in cui sono comunque preponderanti, come si è appena detto, $\mathrm{i}$ ricordi elaborati durante le vacanze estive. ${ }^{3}$ L'epoca concreta del ritorno è, presumibilmente, assai vicina a quella della scrittura, ossia il 1948-1949, anni cruciali nella storia del nostro dopoguerra, in cui si rompe il patto di unità delle forze antifasciste che avevano partecipato alla Resistenza.

Da questa piattaforma cronologica, la narrazione si muove, in forma discontinua, con oscillazioni incessanti lungo gli anni precedenti, una ventina, in cui la memoria del paese è ricostruita, come si è visto, attraverso la testimonianza di Nuto, e poi ancora all'indietro, nei primi anni di vita del protagonista fino a quando parte per fare il servizio militare a Genova. ${ }^{4}$

I capitoli in cui è la memoria di Anguilla ad assumere il racconto in prima persona sono naturalmente i più numerosi ed $\mathrm{i}$ più poetici, al punto da sembrare, come si è detto prima, veri poemi in prosa.

Il passato di Anguilla è suddiviso in due epoche, rappresentate dal servizio nella cascina della Gaminella fin verso i 13 anni e poi in quello della Mora, la tenuta in cui vive e lavora fin verso i vent'anni, quando è chiamato al servizio di leva. Due tappe della vita, quindi, l'infanzia e l'adolescenza, sempre vissute nella condizione di «bastardo» al servizio dei padroni. Nell'epoca della Gaminella, in una situazione di maggiori ristrettezze, avviene l'epifania delle cose, la scoperta emozionata del mondo e della natura. La Gaminella è, come sostiene Elio Gioanola, «il luogo attorno a cui si organizza la serie della fissità totale, rispondente alla primissima rivelazione delle cose, quando gli aspetti della natura, gli odori, i rumori, i sapori, si sono radicati nell'antica memoria infantile come figure significanti dell'essere» (Gioanola 2003: 48). Alla Mora invece, in un clima socialmente più confortevole e grazie alla presenza delle figlie del sor Matteo, si schiudono davanti ai suoi occhi il mistero del sesso ed i presagi dell'avventura che lo avrebbe portato lontano dal paese. Anguilla dalla sua postazione di servitore osserva le padroncine Irene e Silvia, mentre diventano donne, alle prese con le prime schermaglie amorose. Mentre lui sgrana gli occhi sul mistero, Nuto, che, con il suo sguardo da gatto, ha già cominciato a conoscere il mondo (gira per le Langhe a suonare il clarino nelle feste paesane), gli fa da fratello maggiore, spiegandogli come vanno le cose. Già «ai tempi della Mora, del lavoro in cascina, lui che ha tre anni piú di me sapeva [..] fischiare e suonare la chitarra, era cercato e ascoltato, ragionava coi grandi, con noi ragazzi, strizzava l'occhio alle donne» (Pavese 2000: 786).

Il passato «americano» è appena evocato: soltanto tre dei trentadue capitoli di cui si compone il romanzo, verso i quali la critica si è mostrata poco benevola, ma

\footnotetext{
3 Secondo Ulla Musarra-Schrøder, mentre «nel romanzo di memorie tradizionale l'atto della memoria fa parte della narrazione, ne La luna e i falò la memoria è attiva su un piano intermedio», nel senso che ha bisogno di un «io-mediatore» per essere attivata, come in Proust (Musarra 1989: 157).

4 Per Vittorio Spinazzola «l'andirivieni instancabile tra passato e presente» è il procedimento tipico delle «storie di rimpatrio» (Spinazzola 2001: 126).
} 
che hanno in fondo la funzione «tecnica» di giustificare l'assenza di Anguilla e di creare quindi una lacuna nella sua memoria che richiede l'intervento esplicativo di Nuto. ${ }^{5}$ Nell'autografo, questi capitoli, soprattutto l'undicesimo, sono fra quelli che presentano più cancellature, indizio evidente di una stesura più incerta. ${ }^{6}$

Il loro carattere funzionale è provato inoltre, come ha precisato Van Den Bossche dalla presenza di prolessi, del tipo «sapevo già che sarei tornato» che preannunciano periodicamente il rientro del protagonista (Van Den Bossche, 1989: 726). Lì in America, persino la luna sembra aver cambiato i connotati: la notte che trascorre con il camioncino in panne nel deserto nei pressi di Yuma, la luna che spunta tra le nuvole gli sembra una «ferita di coltello [che] insanguinava la pianura».

Nonostante siano ricordi di «seconda mano», costruiti con ambienti ed atmosfere che l'autore, che, com'è noto, non riuscì mai ad andare in America, prese in prestito dagli autori che amava, risultano, a mio avviso, credibili e suggestivi.

Le memorie si affollano lungo il percorso di Anguilla attraverso le diverse stagioni del suo passato: a volte è un odore a fare scattare il ricordo, altre volte l'immagine concreta di un oggetto, la visione di una cascina, di un falò, della vigna o del tronco su cui si sedeva da ragazzo a chiacchierare con Nuto. ${ }^{7}$ Il congegno è quello della libera associazione, come confermano le parole di Anguilla quando ammette che «tutto quello che per tanti anni ti sei portato dentro senza saperlo si sveglia adesso al tintinnio di una martinicca, al colpo di coda di un bue, al gusto di una minestra, a una voce che senti sulla piazza di notte» (Pavese 2000: 813).

La prima sensazione che gli porta il passato è che in fondo nulla è cambiato da allora, che tutto è rimasto uguale. ${ }^{8}$ È questa la sensazione rassicurante che riceve appena arrivato in paese: «Stessi rumori, stesso vino, stesse facce di una volta» (Pavese 2000: 785). Anche nel salire i gradini della Gaminella, sempre attraverso la ripetizione dell'aggettivo "stesso", Anguilla compone una specie di litania della fissità: «Seguitai a salire, e vidi il portico, il tronco del fico, un rastrello appoggiato all'uscio - la stessa corda col nodo pendeva dal foro dell'uscio. La stessa macchia di verderame intorno alla spalliera sul muro. La stessa pianta di rosmarino sull'angolo della casa» (Pavese 2000: 797). Questo processo di stasi del tempo che pare essersi fermato all'epoca della Gaminella dovrebbe rassicurare Anguilla: in fondo chiunque visita i luoghi in cui è cresciuto ha l'illusione segreta di ritrovarli intatti come in un presepe. Le cose si presentano ad Anguilla con il nitore e la verginità di

${ }^{5}$ Secondo Marziano Guglielminetti, Anguilla potrebbe sottoscrivere ciò che aveva appuntato Pavese nel suo diario, il 10 luglio 1947: «Non ti piacciono le storie partigiane o terroristiche, sono troppo spiegabili», per cui l'ingrato compito di raccontarle ne La luna e i falò è ceduto all'amico Nuto (Guglielminetti 2000: LX). Una frase di Pinolo Scaglione riprodotta in Fumatori di carta la dice lunga sul disagio militante dell'amico: «Riguardo alla resistenza dico solo questo. A tutti quelli che lo hanno conosciuto, ma veramente conosciuto, chi di costoro può immaginare Pavese con un mitra in mano?» (Vaccaneo 1999: 192).

${ }^{6}$ Elisabetta Soletti, nella Nota linguistica: «Una notevole incertezza di impostazione, di taglio e di scrittura mostrano infine gli inserti americani, in particolare il capitolo XI» (Pavese 2000: 1167).

7 Sui «protocolli» mnemonici e rituali del ritorno, come l'enumerazione di luoghi ed oggetti, vedi il saggio di Bart Van Den Bossche, La luna e i falò: una storia senza fine (2001).

${ }^{8}$ Come ha segnalato Gian Luigi Beccaria, «il protagonista torna da lontano per rivedere l'identico [...]» (Beccaria 2005: XI). 
stampi che vede ora, soltanto, per la prima volta. In quella cascina dove ha trascorso l'infanzia, ritrova addirittura un altro se stesso: Cinto, così si chiama il ragazzo che vive adesso alla Gaminella, non è un bastardo com'era lui allora, ma ha comunque le stimmate della miseria e della disperazione. Questa volta il messaggio di immobilità è molto più potente di quello realizzato attraverso l'aggettivo "stesso". Di fronte a Cinto, Anguilla non si limita a rivedere se stesso da ragazzo, ma si identifica con lui, come era successo al personaggio che si rivede bambino contemplando la vigna nel racconto omonimo di Feria d'agosto o a Corrado, il protagonista de La casa in collina che, appoggiando la mano sulla nuca di Dino, ha la sensazione di sentire: «sotto le dita me stesso ragazzo [...]» (Pavese 2000: 410).

[Cinto] - racconta Anguilla - «era zoppo, rachitico, vidi il ginocchio non più grosso del suo braccio, si tirava il piede dietro come un peso. Avrà avuto dieci anni, e vederlo su quell'aia era come vedere me stesso». È come se si aprisse una porta nel tempo da cui si aspetta di veder apparire le figure del passato: «[...] diedi un'occhiata sotto il portico, dietro il fico, alle melighe, se comparissero Angiolina e Giulia» (Pavese 2000: 797). La vertigine del ricordo lo porta addirittura ad invidiare il ragazzo: «Cos'avrei dato per vedere ancora il mondo con gli occhi di Cinto, ricominciare in Gaminella come lui, con quello stesso padre, magari con quella gamba - adesso che sapevo tante cose e sapevo difendermi. Non era mica compassione che provavo per lui, certi momenti lo invidiavo» (Pavese 2000: 847). Cinto però si sottrae subito a quella trama di complicità in cui cerca di irretirlo quello straniero un po' nostalgico, gettando in mezzo a quei ricordi lontani di un passato che non gli appartiene le sequenze tragiche dei cadaveri che cominciano ad apparire sulla collina. L'ostinazione di Cinto ricorda quella di Dino, il fanciullo che nella Casa in collina svia i ricordi di un altro adulto in fuga verso il passato, Corrado, per riportare l'attenzione sul presente e sulla guerra.

Ad accrescere la percezione di immobilità che avverte il protagonista contribuisce anche, come ha rilevato Elio Gioanola, la costellazione di toponimi che si ripetono come una giaculatoria, «senza alcuna necessità funzionale» (Gioanola, 2003: 50). Si pensi alla prima pagina del romanzo con la costellazione mitica della toponimia delle Langhe: Canelli, Barbaresco, Alba, Monticello, Neive e Cravanzana.

Anche il tempo sembra rallentare il proprio corso in quanto non si misura con gli anni ma con le stagioni che si ripetono immutabili per l'eternità. «Il bello di quei tempi - dice Anguilla - era che tutto si faceva a stagione [...]». Le date e le altre coordinate temporali sono sostituite da categorie che hanno le qualità poetiche da Leopardi attribuite al vago e all'indefinito e che qui hanno la funzione di suggerire un tempo statico. Ecco un campionario delle più rappresentative: «l'anno che grandinò», «l'autunno prima della grossa grandine», «già al tempo di una delle prime vendemmie», «quel settembre», «in quei giorni», «quella primavera», «quell'inverno», «una domenica d'estate»?.

9 «Il tempo della «Mora» - sostiene Gioanola - è tutto scandito dal riferimento alle stagioni: mai un accenno agli anni o ad altro termine cronologico convenzionale; è una pseudo-cronologia che, mentre sembra alludere continuamente al passare del tempo, in realtà rimanda alla figura del cerchio, dove il movimento ritorna su se stesso perpetuamente» (Gioanola 1969: 61). 
La coscienza della immutabilità delle cose che mantengono la purezza e l'integrità della prima volta in cui sono state viste, non è solo un ingranaggio della memoria, ma anche il requisito estetico del mito. Pavese, come si sa, ha dedicato al mito molte pagine della sua produzione: mito è l'involucro magico che racchiude le cose viste per la prima volta, mito è, dice lo stesso scrittore, «un fatto avvenuto una volta per tutte».

Dopo questo primo movimento in cui la memoria sembra avvertire l'immobilità del passato, gli oggetti vengono riconosciuti e decifrati in quel processo conoscitivo che abbiamo già illustrato e che potremmo riassumere sinteticamente nella formula «ricordare- = capire». Il processo della conoscenza è introdotto dall'avverbio «adesso» che insieme ai verbi «capire» e «sapere» compone un'altra specie di litania che accompagna le diverse tappe del ritorno di Anguilla. Costui sembra quasi trasformarsi in Renzo Tramaglino quando, nell'epilogo de I promessi sposi, elenca, secondo una delle regole imprescindibili del romanzo di formazione, tutte le cose che ha imparato dalle sue disavventure. Con la differenza però che questo di Pavese, come ha giustamente individuato María de las Nieves Muñiz, è un romanzo di formazione «à rebours», cioè a ritroso, perché il protagonista cresce e matura procedendo all'indietro, verso il passato, non verso il futuro (Muñiz Muñiz 1990: 186).

Ciò che «adesso» capisce è la miseria in cui ha vissuto («Adesso sapevo che eravamo dei miserabili»), i riti rusticani del sesso («adesso sapevo [...] perché uomini e donne, giovanotti impomatati e figliole superbe, si scontravano, si prendevano, si ridevano in faccia e ballavano insieme»»)), le inquietudini delle figlie del sor Matteo ed anche i loro limiti («adesso [...] ho capito che le figlie del sor Matteo non erano poi le più belle. [...] Ho anche capito che non erano in gamba, che col loro pianoforte, coi romanzi, col tè, coi parasoli, non sapevano farsi una vita, essere vere signore, dominare un uomo e una casa») (Pavese 2000: 782, 785, 856).

Come l'Anguilla-bambino della Gaminella, anche 1'Anguilla-adolescente della Mora è immaginato retroattivamente con lo sguardo già proiettato verso il futuro. Come un riflesso di coscienza che procede a ritroso nel tempo ed è ricevuto, nel passato, da uno specchio che lo rimanda un'altra volta verso il futuro: «Capivo che da ragazzo, anche quando facevo correre la capra, quando d'inverno rompevo con rabbia le fascine mettendoci il piede sopra [...] anche allora mi preparavo al mio destino, a vivere senza una casa, a sperare che di là delle colline ci fosse un paese più bello e più ricco» (Pavese 2000: 806).

L'infanzia verso cui tende, retroattivamente, il protagonista per maturare non è quindi soltanto quella inoffensiva degli archetipi e dello stupore. Come ha efficacemente sottolineato María de las N. Muñiz, «il bambino più autenticamente pavesiano non è perciò quello che vive ancora primitivamente a contatto diretto con le cose, ma quello che già prefigura l'avvenire e vede nell'adulto un simbolo enigmatico del proprio futuro» (Muñiz Muñiz 1990: 193).

La liturgia della comprensione si trasforma quindi in un andirivieni vertiginoso, in cui l'avverbio della conoscenza, «adesso», con la sua carica semantica di contemporaneità, si sposta a volte, o meglio, rimbalza, in una zona del passato che non sarebbe sintatticamente di sua competenza. È il caso, ad esempio, di un «Adesso» pronunciato da Anguilla quarantenne, e che viene riferito al momento in cui Nuto va 
soldato e lui è ancora un adolescente al servizio nella Mora: «io adesso ero un uomo e non succedeva più che il massaro mi menasse una cinghiata o qualcuno mi dicesse bastardo». «Adesso - ribadisce ancora più avanti - sapevo che cos'era una donna, sapevo perché la musica sui balli mi metteva voglia di girare le campagne come i cani» (Pavese 2000: 878).

Ma, alla fine, l'illusione della perdurabilità ha breve durata. Nel sistema della memoria si apre una falla nella quale cominciano ad affiorare i cadaveri del passato. Il passato è un cimitero, come il villaggio di Comala in cui Juan Preciado, nel romanzo di Juan Rulfo, è andato per ritrovare suo padre, Pedro Páramo. Sono tutti morti, quelli della Gaminella e della Mora, il sor Matteo e le sue figlie, Irene («così bionda»), Silvia («che faceva ancora le scale di corsa») e Santina (che «andava a letto con le brigate nere»), tutti coloro che sperava di ritrovare e di essere da loro riconosciuto: «Ero tornato, ero sbucato, avevo fatto fortuna [...] ma le facce, le voci e le mani che dovevano toccarmi e riconoscermi, non c'erano più. Da un pezzo non c'erano più $^{10}$. Quel che restava era come una piazza l'indomani della fiera, una vigna dopo la vendemmia, il tornar solo in trattoria quando qualcuno ti ha piantato» (Pavese 2000: 828). La sconfitta implicita nel ritorno e nella strategia della memoria che esso ha innescato è espressa magistralmente in un formula che riassume tutto ciò che ancora gli restava da capire: «Non sapevo che crescere vuol dire andarsene, invecchiare, veder morire, ritrovare la Mora com'era adesso» (Pavese 2000: 829). Il passato è un cumulo di macerie: persino la Gaminella, il santuario della sua infanzia, viene distrutta da un incendio proprio davanti ai suoi occhi.

Come se non bastassero i caduti della vita, cominciano ad apparire anche quelli della guerra. La collina, in cui erano stati frettolosamente sepolti, non ce la fa più a trattenerli e li fa affiorare, forse li vomita, con le tracce ancora visibili delle torture e delle esecuzioni sommarie con cui erano stati liquidati. A poco a poco appaiono cadaveri di fascisti e di partigiani uccisi nelle ultime fasi della resistenza.

L'incendio della Gaminella segna anche la degenerazione dei fuochi (i falò del titolo) festivi o rituali che hanno sin qui illuminato il romanzo, in simboli di distruzione e di morte. Anche il fuoco segue così il percorso della memoria : all'inizio è un elemento rappresentativo del passato con funzioni rituali (ingrassano la terra) e festive (i fuochi di San Giovanni) e poi, come la memoria, diventa veicolo di morte.

Pavese conosceva bene la liturgia del fuoco, sia perché aveva vissuto in campagna ed aveva visto e goduto dei fuochi di San Giovanni, sia perché si era interessato al ruolo che il fuoco aveva avuto presso le civiltà primitive. Una delle sue letture formative era stata Il ramo d'oro. Studio sulla magia e la religione dell'antropologo inglese James George Frazer, che aveva fatto tradurre per una collana scientifica dell'Einaudi. Nel saggio si analizzano i miti ed i riti legati al fuoco. Alle origini si trattava di sacrifici umani, in quanto venivano bruciati degli esseri viventi ed avevano oltre che funzioni taumaturgiche anche quella di assicurare la fertilità della terra. Tradizione che è rimasta, anche se incruenta, nella cultura contadina come

10 Nel Mestiere di vivere, in un appunto dell'8 febbraio 1949, Pavese sembrava preannunciare la frustrazione del ritorno: «Perché la gloria venga gradita devono resuscitare i morti, ringiovanire vecchi, tornare lontani» (Pavese 2000: 363). 
dimostrano le parole di Cinto quando spiega ad Anguilla che il fuoco «fa bene alle campagne». Anche Nuto conferma che i fuochi «svegliano la terra». I falò sono quindi la versione civilizzata dei sacrifici umani che una volta servivano a rendere fertile la terra o ad invocare la pioggia.

Con l'incendio della Gaminella è come se il fuoco recuperasse il valore ancestrale. Quell'incendio è in realtà un sacrificio umano perché Valino, subito dopo aver dato sfogo alla sua rabbia bestiale contro la miseria che lo perseguita, si impicca ad un albero del cortile.

L'ultimo falò è quello che consuma il corpo senza vita di Santina, la figlia più piccola del sor Matteo, che viene fucilata dai partigiani con l'accusa di fare il doppio gioco. È Nuto a raccontare come sono andate le cose: «Ci pensò Baracca. Fece tagliare tanto sarmento nella vigna e la coprimmo fin che bastò. Poi ci versammo la benzina e demmo fuoco. A mezzogiorno era tutta cenere. L'altr'anno c'era ancora il segno, come il letto di un falò» (Pavese 2000: 896).

La morte di Valino, di Santina, dei fascisti e dei partigiani dissotterrati dalla collina e degli altri innumerevoli caduti che spopolano il passato di Anguilla dimostra come Pavese fosse convinto che la vita, come una divinità cruenta, continuasse ad esigere dei sacrifici umani. E fra i predestinati ad essere immolati, c'era purtroppo anche lui, come già lasciano presagire le parole della dedica all'amico Pinolo e le riflessioni che andava trascrivendo nel Mestiere di vivere.

\section{Bibliografia}

BECCARIA, Gian Luigi (2005): «Introduzione» ne La luna e i falò. Torino, Einaudi, pp. v-xxxiii.

Calvino, Italo (2007): Pavese e i sacrifici umani in Cesare Pavese, Saggi 1945-1985. Milano, Mondadori, t. 2, pp. 245-252.

FRAZER, James Geroge (2007): Il ramo d'oro. Studio sulla magia e la religione. Torino, Bollati Boringhieri.

GIOANOLA, Elio (1969): «L'essere e la morte ne La luna e i falo», in Sigma, n. 22, pp. 5166.

GiOANOLA, Elio (2003): Cesare Pavese. La realtà, l'altrove, il silenzio. Milano, Jaca Book. GIOVANARDI, Stefano (1996): La luna e i falo, in AA. VV., Letteratura italiana, Le opere. Torino. Einaudi, IV, pp. 630-646.

GuglielminetTI, Marziano (2000): «Cesare Pavese romanziere», in Pavese. Tutti i roman$z i$. Torino, Einaudi, pp. ix-lxvi.

LEOPARDI, Giacomo (1991): Zibaldone di pensieri, Edizione critica e annotata a cura di Giuseppe Pacella. Milano, Garzanti.

MUÑIZ MuÑIZ, $\mathrm{M}^{\mathrm{a}}$ de las Nieves (1990): «Cesare Pavese: ritorno en abîme», in Poetiche della temporalità. Palermo, Palumbo, pp. 185-199.

MUSARRA-SCHRØDER, Ulla (1989): «Il rinnovamento della ricerca proustiana: Pavese, Butor, Nabokov», in Narciso e lo specchio. Il romanzo moderno in prima persona. Roma, Bulzoni.

PAVESE, Cesare (1968): Feria d'agosto. Torino, Einaudi. 
PAVESE, Cesare (1968): Lettere 1926-1950, voll. II, a cura di Lorenzo Mondo e Italo Calvino. Torino, Einaudi.

PAVESE, Cesare (1968): Lavorare stanca. Torino, Einaudi.

PAVESE, Cesare (1999): Dialoghi con Leucò, a cura di Sergio Givone. Torino, Einaudi.

PAVESE, Cesare (2000): Il mestiere di vivere. Diario 1935-1950, a cura di Marziano Guglielminetti e Laura Nay; "introduzione" di Cesare Segre. Torino, Einaudi.

PAVESE, Cesare (2000): Tutti i romanzi, a cura di Marziano Guglielminetti. Torino, Einaudi.

Rulfo, Juan (1955): Pedro Páramo. México, Fondo de Cultura Económica.

SPINAZZOLA, Vittorio (2001): «Nelle Langhe, dopo la guerra», in Itaca addio. Milano, Il Saggiatore, pp. 89-137.

VACCANEO, Franco (1999): Fumatori di carta. Nuto e Pavese. Torino, Omega Edizioni.

VAN DEN Bossche, Bart (1989): «La temporalità ne La luna e i falò» in Critica letteraria, XVIII, n. 65, pp. 721-738.

VAN DEN BOSSCHE, Bart (2001): «La luna e i falò: una storia senza fine», in «Nulla è veramente accaduto». Strategie discorsive del mito nell'opera di Cesare Pavese. Firenze, Leuven University Press- Franco Cesati Editore. 